Marchioli, A., (2006), « Marketing social et efficacité des campagnes de prévention de santé publique : apports et implications des récents modèles de la communication persuasive », Communication \& Marketing (anciennement Market Management, dirigé par Y. Chirouze), 1, pp. 17-36 


\title{
Marketing social et efficacité des campagnes de prévention de santé publique : apports et implications des récents modèles de la communication persuasive.
}

\author{
Audrey MARCHIOLI ${ }^{1}$
}

Résumé - Illustrant un exemple de communication persuasive appliquée au marketing, l'article montre comment les recherches en communication de santé publique peuvent aider le marketing social à changer les comportements en matière de santé et accroître l'efficacité des campagnes. Suivant un double objectif scientifique et opérationnel, l'article envisage, après une présentation des récents modèles persuasifs, leurs apports au marketing social ainsi que des implications pratiques utiles aux praticiens.

Mots clés - Marketing social, persuasion, communication de santé publique, changement de comportement, appels à la peur, communication engageante.

\begin{abstract}
Illustrating an example of persuasive communication applied to marketing, the article shows how research in public health communication can help social marketing to change health behaviors and increase campaign's effectiveness. According to a both scientific and operational objective, the article considers, after a presentation of the recent persuasive models, their contributions to social marketing as well as useful implications for practitioners.
\end{abstract}

Key words - Social marketing, persuasion, public health communication, behavior change, fear appeals, commitment.

1 Laboratoire de recherche en SIC I3M «Informations, milieux, médias, médiations », courriel audrey.marchioli@unice.fr. 
Le savoir-faire et les techniques du marketing ne se limitent pas aux entreprises, ils peuvent également s'étendre au domaine social. Le marketing prend alors l'appellation de marketing social. En utilisant les principes et les techniques du marketing, il vise à influencer un public-cible afin qu'il accepte, rejette, modifie ou abandonne volontairement un comportement bénéficiant à un individu, à un groupe ou à la société dans son ensemble (Kotler et al. 2002). Le marketing social permet de donner aux praticiens un cadre d'action pour résoudre les problèmes sociaux et changer les comportements (Gallopel, 2003). Dans le domaine de la santé, les pouvoirs publics, tels l'État, les ministères ou les collectivités territoriales, ont souvent recours au savoir-faire du marketing social pour l'élaboration de campagnes de santé publique, encore appelées campagnes de prévention. Celles-ci utilisent les médias de masse et recouvrent de nombreux domaines comme la lutte contre l'alcoolisme, le tabagisme, les maladies sexuellement transmissibles ou encore la prévention du cancer. L'objectif principal des campagnes de marketing social dans le domaine de la santé est de créer, renforcer ou modifier certaines attitudes afin qu'elles génèrent des comportements davantage bénéfiques pour la santé (Courbet, 2003). Les professionnels du marketing social sont donc confrontés aux problèmes suivant : Comment concevoir et mettre en œuvre des campagnes efficaces ? Comment amener un individu à changer ses comportements en matière de santé et à adopter un comportement plus sécuritaire ? Comment augmenter l'impact des campagnes ? Pour répondre à ces questions, le marketing social propose une démarche en neuf étapes (Kotler et al, 2002 ; Gallopel, 2003) comprenant : l'analyse du problème social contre lequel le praticien veut lutter (e.g., la prévention du tabagisme), le choix de la cible d'action prioritaire (e.g., les jeunes fumeurs), la fixation des objectifs (e.g., inciter les jeunes à arrêter de fumer), la connaissance de la cible (e.g., croyances, attitudes, valeurs, freins, motivations à l'égard du tabac), le choix d'un concept de campagne, le plan de campagne (e.g., campagne télévisée de prévention du tabagisme), le pré-testage de la campagne, l'application et enfin l'évaluation des résultats.

Afin d'atteindre ses objectifs et accroître l'efficacité de sa démarche, le marketing social peut intégrer les recherches en communication persuasive de santé publique. En suivant la conception de la communication persuasive de Chabrol et al., (2004), la communication persuasive de santé publique inscrit son objet d'étude au sein d'un système d'échanges socio-économiques et sociopolitiques à multiples enjeux, dans lequel une organisation (i.e., les pouvoirs publics) dotée d'intentionnalités, cherche à orienter les comportements des publics (i.e., les citoyens ) également dotés d'intentionnalités, de manière à ce que ces comportements contribuent à réaliser les objectifs de l'organisation (i.e., changer les comportements néfastes pour la santé). Grâce à des moyens de communication de masse, les pouvoirs publics exposent les citoyens à des dispositifs capables de produire les effets escomptés. Les citoyens-récepteurs qui, au sein d'un contexte socio-physico- 
temporel de réception, sont en contact sensoriel avec ces dispositifs, mettent en œuvre des traitements conduisant, dans certaines conditions, à la formation, au renforcement ou au changement de leurs représentations cognitives ou affectives (attitudes) et parfois de leurs comportements.

Au regard de cette définition, nous voyons que le marketing social et la communication persuasive de santé publique poursuivent un même objectif : changer les comportements de santé ${ }^{2}$. C'est pourquoi la communication de santé publique et principalement les recherches sur la réception et l'influence peuvent être d'un apport précieux au marketing social pour accroître l'efficacité des campagnes. Ces recherches, ayant pour cadre disciplinaire la psychologie sociale et cognitive, visent à identifier, d'une part, les processus psychologiques de traitement des campagnes et leurs effets sur le changement de comportement et, d'autre part, les facteurs pouvant influer sur ces traitements. L'objectif de cet article sera donc, d'une part, de présenter les modèles actuels du changement de comportements en matière de santé, issus des recherches sur la réception de la communication persuasive de santé publique, et, d'autre part, identifier leurs apports au marketing social. Afin d'intéresser scientifiques et professionnels, l'article articulera apports théoriques et implications pratiques. Nous présentons, dans un premier point, les modèles généraux de la communication de santé publique envisageant le changement de comportement en matière de santé. Dans un deuxième point, nous nous pencherons sur les modèles analysant l'effet des campagnes générant des affects négatifs ou «appels à la peur » sur le changement de comportement. Dans un troisième point, nous mettrons en évidence une nouvelle forme de communication persuasive de santé publique : la communication engageante et nous considérerons ses implications pour le marketing social. Enfin, dans un dernier point, nous présenterons quelques conseils, issus des recherches présentées, utiles aux praticiens du marketing social pour la conception de campagnes efficaces.

\section{Persuasion et COMmunication de SANTe PUBLIQUE : Les MOdèles Cognitifs du} CHANGEMENT DE COMPORTEMENT

Les modèles actuels en communication de santé publique envisageant les effets des campagnes sur le changement de comportement sont issus du paradigme de la cognition et des modèles duaux du traitement de l'information (Chaiken et Trope, 1999). Ils s'attachent à mettre en évidence les

\footnotetext{
${ }^{2}$ La santé est prise, ici, dans son sens le plus large Le terme de «comportement de santé » est emprunté à la psychologie de la santé. Il désigne «des actions faites par un individu et ayant une influence positive ou négative sur sa santé » (Godin, 2002, p. 375). Les comportements de santé peuvent être, par exemple, conduire sa voiture sous l'influence de l'alcool, se brosser les dents, fumer la cigarette, utiliser des préservatifs lors de relations sexuelles à risque.
} 
processus psychologiques mis en route dans le traitement des campagnes et responsables du changement de comportement. Après une présentation des principaux modèles, nous envisageons leurs implications pour le marketing social.

\subsection{Des traitements au changement d'attitude}

Les modèles des doubles processus de la persuasion - le modèle de la probabilité d'élaboration (Elaboration Likelihood Model, ELM, Petty et Cacioppo, 1986; Petty et Wegener, 1999) et le modèle heuristique-systématique (Heuristic-Systematic Model, HSM, Chaiken et al. 1989 ; Chen et Chaiken, 1999) ${ }^{3}$ - sont fondamentaux en communication persuasive de santé publique. Adoptant une approche cognitive de la persuasion, ils considèrent le récepteur comme actif dans les traitements. Pour eux, le changement de comportement ne résulte non pas du souvenir que l'individu possède des arguments du message, mais de la production de réponses cognitives (i.e., pensées favorables ou défavorables générées lors de la lecture du message). Pour ces modèles, un récepteur peut traiter un message persuasif en empruntant, soit, la voie centrale (ELM) / systématique (HSM), soit, la voie périphérique (ELM) / heuristique (HSM). Ils s'accordent sur le fait que l'emprunt d'une voie dépend de la motivation des récepteurs, de leur implication et de leur capacité à traiter les arguments du message. Pour l'ELM, c'est l'élaboration qui médiatise la persuasion. L'élaboration est «l'importance avec laquelle le récepteur pense aux arguments contenus dans le message » (Petty et Cacioppo, 1986, p. 128). En revanche, pour le HSM, ce sont les principes de moindre effort et de suffisance qui médiatisent la persuasion. Le récepteur traite le message en cherchant un équilibre entre un minimum d'efforts cognitifs et la satisfaction de ses motivations (Chen et Chaiken, 1999). Ainsi, lorsqu'un individu dispose d'une motivation et d'une capacité importantes pour traiter le contenu du message persuasif ou lorsque l'équilibre est faible, il emprunte la voie centrale (ELM) / systématique (HSM). Il forme son jugement sur la qualité de l'argumentation. L'attitude formée à l'issue de ce traitement est stable, résistante à une tentative de contre-persuasion et fortement prédictive du comportement. En revanche, si le récepteur dispose d'une motivation et d'une capacité faibles de traitement du contenu persuasif ou que l'équilibre est important, il emprunte la voie périphérique (ELM) / heuristique (HSM). Pour l'ELM, il base son jugement sur des indices périphériques comme des indices contextuels (e.g. longueur du message) ou des indices propres à la source du message (e.g. crédibilité, expertise, sympathie, attirance physique) (Petty et Wegener, 1999). Pour le HSM, le récepteur base son jugement sur des heuristiques qui sont des structures de connaissances stockées en mémoire et facilement accessibles

\footnotetext{
${ }^{3}$ Notons que nous ne présentons pas les modèles dans leur intégralité mais seulement les points qui servent directement notre propos. Pour une présentation complète et récente se référer à Corneille (1993), Meyer (2000), Petty et Wegener (1999), Chen et Chaiken (1999).
} 
(e.g. « on peut faire confiance aux experts ») (Chen et Chaiken, 1999). Qu'il traite le message sur la base d'heuristiques ou d'indices périphériques, le sujet fourni peu d'effort cognitif. L'attitude formée est instable, peu résistante à une tentative de contre-persuasion et peu prédictive du comportement. Objet de nombreuses validations empiriques en communication persuasive de santé publique (e.g. Kreuter et al. 1999 ; Girandola et Atkinson, 2003), ils sont très explicites sur les mécanismes psychologiques sous-jacents au changement d'attitude. Cependant, ils restent au stade de l'intention comportementale et n'envisagent pas le changement effectif de comportement.

\subsection{Du changement d'attitude au comportement de santé}

Les campagnes de marketing social cherchent à modifier les attitudes en matière de santé mais visent également à agir sur les comportements. Les modèles des doubles processus étant peu explicites sur ce point, il y a nécessité de les combiner avec d'autres modèles. En 2000, Rosen suggère une utilisation conjointe de l'ELM et d'un modèle spécifique au comportement de santé : le modèle trans-théorique de Prochaska et al. (1992). Pour ce dernier, en matière de santé, un individu progresserait par cinq stades avant de changer de comportement : la «précontemplation » (i.e., déni du problème et/ou ignorance de la menace), la «contemplation » (i.e., considération du changement), la préparation (i.e., intention de se protéger), l'action (i.e., changement de comportement) et la maintenance du nouveau comportement. Pour Rosen (2000), la motivation à traiter les arguments du message diffère en fonction des stades. Connaître le stade auquel se situe la cible permettrait de prévoir l'effet de la campagne sur les comportements. Griffin et al., (1999) intègrent les apports des modèles duaux et de la théorie du comportement planifié (Ajzen, 1991). Ils proposent un modèle de la communication persuasive de santé publique spécifiant, à la fois, les traitements mis en route, leurs effets sur le changement d'attitude et sur le changement effectif de comportement. Le modèle étudie la relation entre la recherche d'informations sur le risque, son traitement et l'adoption d'un comportement de prévention pour la santé. Pour Griffin et al. (1999), la manière dont le récepteur recherche et traite l'information sur le risque dépend de trois facteurs : la suffisance perçue de l'information (i.e., quantité d'informations dont le récepteur pense avoir besoin pour évaluer un risque), la capacité perçue à chercher et traiter l'information (i.e., la cible se sent-elle capable de rechercher et traiter les informations sur le risque ?) ainsi que la pertinence perçue des sources d'informations. Ces trois facteurs sont eux-mêmes influencés par quatre facteurs : la réaction affective par rapport au risque perçu (e.g. angoisse, inquiétude), les normes subjectives (i.e., pressions sociales quant à l'exécution d'un comportement), les caractéristiques du risque (e.g. risque mortel) et les caractéristiques individuelles (e.g. sexe, âge, PCS, comportement antérieur). Tous ces facteurs sont inter-dépendants. Par exemple, si le risque génère de l'inquiétude 
chez le récepteur, cela peut influer sur la quantité d'informations dont il pense avoir besoin pour faire face au risque. En fonction de ces facteurs, le récepteur peut mettre en œuvre, soit, un traitement systématique ( $s$ 'il est motivé et capable), soit, un traitement heuristique (s'il n'est ni motivé, ni capable). Ces traitements vont l'amener à former une attitude envers le comportement de prévention. Le modèle de Griffin et al. (1999) lie attitude et comportement en se basant sur la théorie du comportement planifié (Ajzen, 1991). L'adoption du comportement de prévention (e.g. arrêter de fumer) est médiatisée par l'intention comportementale (IC) (e.g. intention d'arrêter de fumer). Celle-ci est fonction de trois facteurs. Tout d'abord, l'attitude envers le comportement (e.g. évaluation favorable ou défavorable du récepteur envers l'intention d'arrêter de fumer) dépendant elle-même des croyances sur les conséquences du comportement (e.g. «si j'arrête de fumer, je diminue ma probabilité de développer un cancer ») et de l'évaluation des résultats de ce comportement (e.g. «si j'arrête de fumer, je serais en bonne santé »). Les normes subjectives, ensuite, qui sont fonction des croyances du récepteur sur ce que les membres de sa famille ou de son groupe de référence attendent de lui quant à l'action (e.g. « ma famille et mes amis sont nonfumeurs, ils pensent que je devrais arrêter de fumer ») et de la motivation du récepteur à s'y conformer (e.g. « je veux faire ce que ma famille et mes amis attendent de moi »). Enfin, le contrôle comportemental perçu (i.e., croyances du récepteur par rapport au degré de facilité/difficulté d'accomplir le comportement) qui dépend des croyances de la cible sur le contrôle. Celles-ci peuvent être internes (e.g. informations, habileté) ou externes (e.g. opportunité, dépendance vis-àvis des autres). Griffin et al. (1999) ajoutent que le contrôle comportemental perçu est influencé par les caractéristiques perçues du risque (e.g., auto-efficacité, pertinence personnelle du risque). Le contrôle peut influer sur le comportement par l'intermédiaire de l'IC ou de manière directe. Objet de nombreuses validations empiriques en prévention pour la santé (e.g. Albarracín et al. 2001 ; Higgins et Conner, 2003), la théorie du comportement planifié s'avère valable pour prédire l'effet des campagnes sur le changement de comportement.

Un nouvel éclairage sur la relation attitude-comportement de santé résiderait dans l'application du modèle MODE (Schuette et Fazio, 1995). Il postule de l'existence de deux processus agissant sur les attitudes et les comportements. Face à une campagne de marketing social (e.g. prévention du tabagisme), la cible (e.g. les fumeurs) utiliserait deux processus pour former son jugement (e.g. jugement sur les risques du tabac). Tout d'abord, via un processus contrôlé, elle utiliserait les informations incluent dans la campagne. Ensuite, via un processus automatique, elle utiliserait son savoir existant stocké en mémoire (e.g. connaissances sur les risques du tabac). L'association attitude-comportement dépend de l'accessibilité. Plus l'attitude formée est accessible, plus elle sera prédictive du comportement. L'accessibilité est opérationnalisée à l'aide des temps de réponse : 
plus le temps de réponse entre l'exposition au message de prévention et l'évaluation de la menace du message (e.g. dangereux) est court, plus l'association est forte chez le récepteur, et plus l'attitude est prédictive du comportement. Ce concept très utilisé en publicité (e.g. Courbet, 2004), n'a à notre connaissance, pas donné d'application en communication persuasive de santé publique. Une telle application peut être intéressante pour l'étude de l'impact des campagnes de prévention sur les comportements.

\subsection{Implications pour le marketing social}

Les modèles de la communication persuasive de santé publique possèdent de nombreuses implications pour le marketing social, notamment pour la connaissance et la segmentation de la cible. Les modèles duaux de la persuasion montrent l'importance de prendre en compte la motivation, l'implication et la capacité de traitement du message de la cible pour que la campagne soit efficace. Le HSM introduit deux indices supplémentaires: la quantité et la qualité des connaissances de la cible sur le thème de la campagne. Plus elle possède de connaissances sur un thème (e.g. connaissances sur les conséquences du tabac sur la santé), plus elle a tendance à adopter un traitement systématique, et à changer ses comportements. Le HSM affine la conception des motivations en proposant trois types de motivations (i.e., motivation à l'exactitude, motivation à la défense, motivation d'impression) (Chaiken et al. 1996). C'est la motivation à la défense (i.e., désir de maintenir des attitudes, croyances et jugements qui soient congruents avec des intérêts matériels ou avec le soi) qui influe le plus dans le processus de persuasion des campagnes de prévention de santé publique. Lorsqu'une campagne génère une menace remettant en cause l'identité, les valeurs et croyances de la cible, cette dernière, si elle est impliquée, va chercher à se protéger de la menace en mettant en place une motivation à la défense. Celle-ci biaisera le traitement systématique en favorisant une contre-argumentation et la résistance la persuasion. Les professionnels du marketing social devront étudier les valeurs, identités et croyances de la cible, et veiller à créer des campagnes ne les remettant pas en cause, sous peine d'un échec. Les modèles liant attitude et comportement introduisent des variables spécifiques à la prévention de santé. Ainsi, dans la connaissance et la segmentation de la cible, les professionnels du marketing social veilleront à prendre en compte des indices psychologiques comme la capacité de la cible à chercher et traiter l'information sur le risque, la vulnérabilité perçue, le risque perçu, les normes subjectives ou encore le contrôle perçu. Il est également intéressant d'identifier à quel stade du modèle de Prochaska et al. (1992) (e.g., précontemplation, contemplation) se situe la cible, afin de créer des messages en conséquence et d'optimiser la persuasion. 


\section{APPELS LA PEUR ET COMMUNICATION PERSUASIVE DE SANTE PUBLIQUE}

Depuis plusieurs années, les campagnes de marketing social pour la prévention de la santé n'hésitent plus à utiliser des images choquantes pour inciter les citoyens à adopter des comportements plus salutaires. Cette pratique, initiée par les pays anglo-saxons il y a une vingtaine d'années, s'est peu à peu développée en France. Ces messages persuasifs appelés «appels à la peur ${ }^{4}$ visent à éveiller, chez le récepteur (e.g. un fumeur), un sentiment de peur en présentant une menace grave et personnellement pertinente (e.g. les fumeurs peuvent contracter un cancer des poumons qui est une maladie mortelle) et en utilisant une représentation concrète et réaliste du danger (e.g. images de fumeurs décédés à la suite d'un cancer, d'organes atteints par le cancer). Ils comportent toujours des recommandations destinées à diminuer la peur ressentie en indiquant des moyens de se protéger de la menace (e.g. «arrêter de fumer vous permettra de vous protéger du cancer ») (Ruiter et al. 2001). Les appels à la peur sont très utilisés en marketing social ${ }^{5}$. L'intérêt pour cette discipline est de comprendre comment ils agissent sur les comportements afin d'optimiser leur utilisation dans les campagnes. Nous présentons différents modèles des appels à la peur en communication persuasive issus de la littérature anglo-saxonne, avant de proposer notre propre modèle schématique et d'envisager ses implications en marketing social.

\subsection{Appels à la peur et motivation à la protection}

En 1983, Rogers propose sa théorie de la motivation à la protection «Protection Motivation Theory » (PMT) qui servira de base à tous les autres modèles sur les appels à la peur. En 2004, une version révisée est établie (Arthur et Quester, 2004). Le modèle initial de la motivation à la protection est rationnel. Il postule que le comportement adopté par un individu exposé à un message persuasif émane d'un choix conscient et délibéré suivant une logique coût/bénéfice. Le sujet choisit un comportement lui permettant d'atteindre ses objectifs avec le moins de contraintes. Le comportement émis peut être, soit, adapté à la menace (e.g. arrêter de fumer pour prévenir un cancer) ou mal adapté (e.g. ne pas arrêter de fumer) (Girandola, 2000). Il dépend de l'éveil de la motivation à la protection, c'est-à-dire, de la motivation du sujet à se protéger de la menace. Celleci est, selon le PMT, fonction de deux processus parallèles de traitement. Ainsi, un individu exposé à un message d'appel à la peur (e.g. message contre le tabagisme) évalue, d'une part, la menace générée par le message. Il examine la sévérité de la menace, c'est-à-dire, l'ampleur des risques générés par la menace (e.g. «fumer provoque le cancer qui une maladie mortelle ») et la

\footnotetext{
${ }^{4}$ Traduction proposée pour «fear appeals ».

${ }^{5}$ Un numéro spécial de la revue Psychology \& Marketing (vol. 21, n9, 2004) a d'ailleurs été consacré aux effets des appels à la peur dans les campagnes de marketing social.
} 
vulnérabilité perçue, c'est-à-dire, le degré à partir duquel la menace représente un danger (e.g. «si je continue à fumer, je risque de développer un cancer »). D'autre part, il évalue le coping ${ }^{6}$, c'est-àdire, les moyens d'échapper à la menace. Le récepteur examine l'efficacité des recommandations proposées par le message (e.g. «arrêter de fumer diminue le risque de contracter un cancer ») et sa propre capacité à réaliser ces recommandations, c'est-à-dire, l'auto-efficacité (e.g. «je me sens capable d'arrêter de fumer »). Le coût de l'adoption des recommandations (e.g. « je vais souffrir de l'arrêt du tabac pendant les premiers mois ») et les bénéfices associés à l'adoption d'un comportement mal-adapté (e.g. «fumer me détend ») influent de manière négative sur la motivation à la protection. Le PMT prévoit un éveil de la motivation à la protection si la menace est jugée forte et le coping valable. Le sujet aurait alors l'intention d'émettre des comportements adaptés à la menace (e.g. arrêter de fumer). En revanche, si la menace est jugée forte mais le coping nonvalable, le sujet a de forte chance d'émettre des comportements mal adaptés à la menace (e.g. continuer à fumer).

Le PMT a fait l'objet de nombreuses validations empiriques dans le domaine de la prévention du tabagisme (Sturges et Rogers, 1996) ou de la toxicomanie (Schoenbachler et Whittler, 1996). La principale limite du modèle réside dans le fait qu'il n'accorde à la peur aucun rôle dans le processus de persuasion. De plus, il reste muet quant aux mécanismes conduisant un récepteur à rejeter le message. Pour pallier ces limites, Arthur et Quester (2004) présentent une version révisée du modèle de la motivation à la protection («Revised Protection Motivation Model», RPMM). Pour celui-ci, c'est l'évaluation de la menace (probabilité d'occurrence et sévérité perçue) qui influence indirectement le changement de comportement à travers la peur générée par le message. Si l'évaluation de la menace, génère chez le récepteur, de la peur, alors il est susceptible de modifier son comportement. L'évaluation du coping (efficacité des recommandations et auto-efficacité) influence les réactions face à la peur générée par l'évaluation de la menace. Ainsi, si les sujets perçoivent les recommandations comme faisables et s'ils se sentent capables de les réaliser, la peur générée aura un effet positif sur le changement de comportement. Ce modèle offre l'avantage d'accorder à la peur une place plus importante dans le processus de persuasion.

\subsection{Le modèle étendu des processus parallèles}

Le modèle étendu des processus parallèles («Extended parallel process model », EPPM) (Witte, 1992 ; 1998), est, à ce jour, le modèle dominant dans le champ de la communication persuasive utilisant les appels à la peur. Il spécifie, à la fois, les effets des appels à la peur sur le changement de

\footnotetext{
${ }^{6}$ Nous employons, ici, le terme anglo-saxon de coping. Dans la littérature francophone, il est parfois remplacé par les termes de «stratégie d'ajustement à la menace » ou de « faire-face ».
} 
comportement et le rôle de l'affect négatif dans le processus de persuasion. Adoptant une optique cognitive, l'EPPM distingue deux voies de traitement du message : le contrôle du danger et le contrôle de la peur. L'emprunt d'une voie est fonction de l'évaluation de la menace perçue et de l'efficacité perçue. La menace perçue se compose de deux dimensions : la vulnérabilité perçue et la sévérité perçue. L'efficacité perçue se compose de deux dimensions : l'efficacité des recommandations et l'auto-efficacité. Si le récepteur juge l'efficacité perçue plus forte que la menace perçue (e.g. «je peux facilement lutter contre le danger»), il va s'engager dans la voie cognitive de contrôle du danger. Le modèle prévoit alors que le sujet accepte les recommandations du message et change de comportement. En revanche, si le récepteur juge la menace perçue plus forte que l'efficacité (e.g. «je cours un grave danger mais je ne peux rien faire »), il va s'engager dans la voie de contrôle de la peur. Cette voie essentiellement émotionnelle va l'amener à rejeter les recommandations du message et à développer des stratégies de résistance à la persuasion via des mécanismes de défense (e.g. déni, remise en cause de la crédibilité de la source). Le modèle prévoit un échec du message.

Le modèle a fait l'objet de validations empiriques, notamment, dans le domaine de la prévention des accidents aux passages à niveaux (Witte et Donohue, 2000) ou du cancer de la peau (Stephenson et Witte, 1998). Sa principale limite réside dans le fait qu'il n'est pas très explicite sur une possible action en parallèle des processus de contrôle de la peur et du danger. (Ruiter et al., 2001). Cependant, l'EPPM est d'un apport précieux pour le marketing social, notamment, en permettant d'identifier comment concevoir des campagnes efficaces. Le modèle a d'ailleurs donné lieu au Persuasive Health Messages Framework, un outil d'aide à la conception des messages. Ainsi, pour le modèle, les campagnes de marketing social utilisant les appels à la peur doivent accentuer la menace, et dans un même temps inclure dans les messages des recommandations efficaces et faisables.

\subsection{Modèles duaux de la persuasion et appels à la peur : le traitement central/systématique biaisé}

Les modèles duaux de la persuasion apportent un éclairage supplémentaire aux modèles des appels à la peur en étant plus précis sur les processus de traitement sous-jacents au changement d'attitude. Cependant, leurs versions initiales (cf. supra) ne permettent pas d'expliquer le rôle et l'effet de la peur dans la dynamique persuasive, ce n'est que des années plus tard, que les chercheurs y parviendront avec la mise en évidence du traitement central/systématique biaisé/défensif (Chaiken et al. 1996). Lorsqu'un récepteur, exposé à un message d'appel à la peur, est fortement impliqué et 
qu'il cherche à contrôler sa peur, il peut traiter le contenu argumentaire de manière biaisée. En effet, les sujets fortement impliqués sont plus critiques à l'égard d'arguments menaçants que les sujets faiblement impliqués. Il mettra en route un traitement dont la réponse finale conforte son point de vue initial (Baron et al. 1994). Le modèle prévoit dans ce cas un échec du message, le sujet ne changera pas de comportement et émettra des réponses défensives comme de l'évitement, du déni ou du fatalisme. Le traitement central/systématique biaisé dépend du but de la cible. Si elle a une évaluation réaliste de la menace et de la manière de s'en protéger, elle a recours à un traitement systématique/central objectif. Elle adopte alors les recommandations proposées par le message et modifie ses comportements. En revanche, si la cible est critique, qu'elle se méfie de la menace et qu'elle ne veut pas changer ses habitudes, elle traite le message de manière défensive en empruntant la voie centrale/systématique biaisée. Das et al. (2003) offrent un nouvel éclairage en postulant pour l'existence de deux types de traitements systématiques biaisés : l'un positif, l'autre négatif. Pour ces auteurs, à la lecture du message, le récepteur mène deux évaluations : l'évaluation de la menace et l'évaluation des recommandations. Si le sujet se sent très vulnérable par rapport à la menace, il effectuera un traitement systématique biaisé négatif. Il aura tendance à minimiser le risque et développer des mécanismes de défense (e.g. déni, réactance). Cependant, si le sujet se sent vulnérable, son traitement des recommandations du message sera systématique biaisé mais positif. Pour ces auteurs, il voudrait se rassurer, pour cela il essaierait de faire apparaître les recommandations du message comme efficaces en cherchant des arguments soutenant leur efficacité à se protéger de la menace. Das et al. (2003) postulent que ce traitement systématique biaisé positif des recommandations optimiserait la persuasion. Analyser les appels à la peur au travers des modèles duaux permet d'affiner le rôle de la peur dans les traitements et son effet sur le changement de comportement.

\subsection{Vers un modèle schématique des appels à la peur en communication persuasive de santé publique : implications pour le marketing social}

Nous proposons un modèle schématique (Figure 1) croisant les modèles duaux de la persuasion et les modèles des appels à la peur (Rogers, 1983 ; Witte, 1998). Ce modèle suppose qu'une campagne utilisant les appels à la peur contienne une menace forte et des recommandations efficaces et faisables. Le récepteur peut traiter le message de trois manières différentes en fonction de sa motivation, de son implication et des buts qu'il poursuit. Si le sujet possède une motivation et une implication faibles, il empruntera la voie périphérique/heuristique. Il basera son jugement sur des indices périphériques et/ou heuristiques. Le modèle prévoit un échec de la campagne. En revanche, si le sujet possède une motivation et une implication fortes, il pourra emprunter la voie de 
traitement centrale/systématique. S'il est critique, méfiant et qu'il juge l'efficacité des recommandations insuffisante pour se protéger de la menace, il traitera le message suivant la voie systématique défensive/biaisée. Le sujet entrera dans un traitement émotionnel destiné à se défendre contre la menace et à contrôler sa peur. Pour cela, il développera des mécanismes de défense (e.g. minimisation de la menace, déni) et ne changera pas de comportement. Si le sujet est réaliste, non critique et qu'il juge les recommandations efficaces pour se protéger de la menace, il traitera le message de manière systématique objective. Il traitera le message de manière cognitive, entrera dans le processus de contrôle du danger et sera motivé à se protéger. Il formera des attitudes positives par rapport aux recommandations et un comportement adapté. Les professionnels du marketing social peuvent utiliser de manière efficiente les appels à la peur en respectant certaines conditions. Ils doivent veiller à sélectionner une cible motivée, impliquée et réaliste vis-à-vis de la menace. Les campagnes peuvent générer une forte menace à condition d'être accompagnées de recommandations efficaces et faisables afin de diminuer la vulnérabilité de la cible et d'augmenter son sentiment d'auto-efficacité.
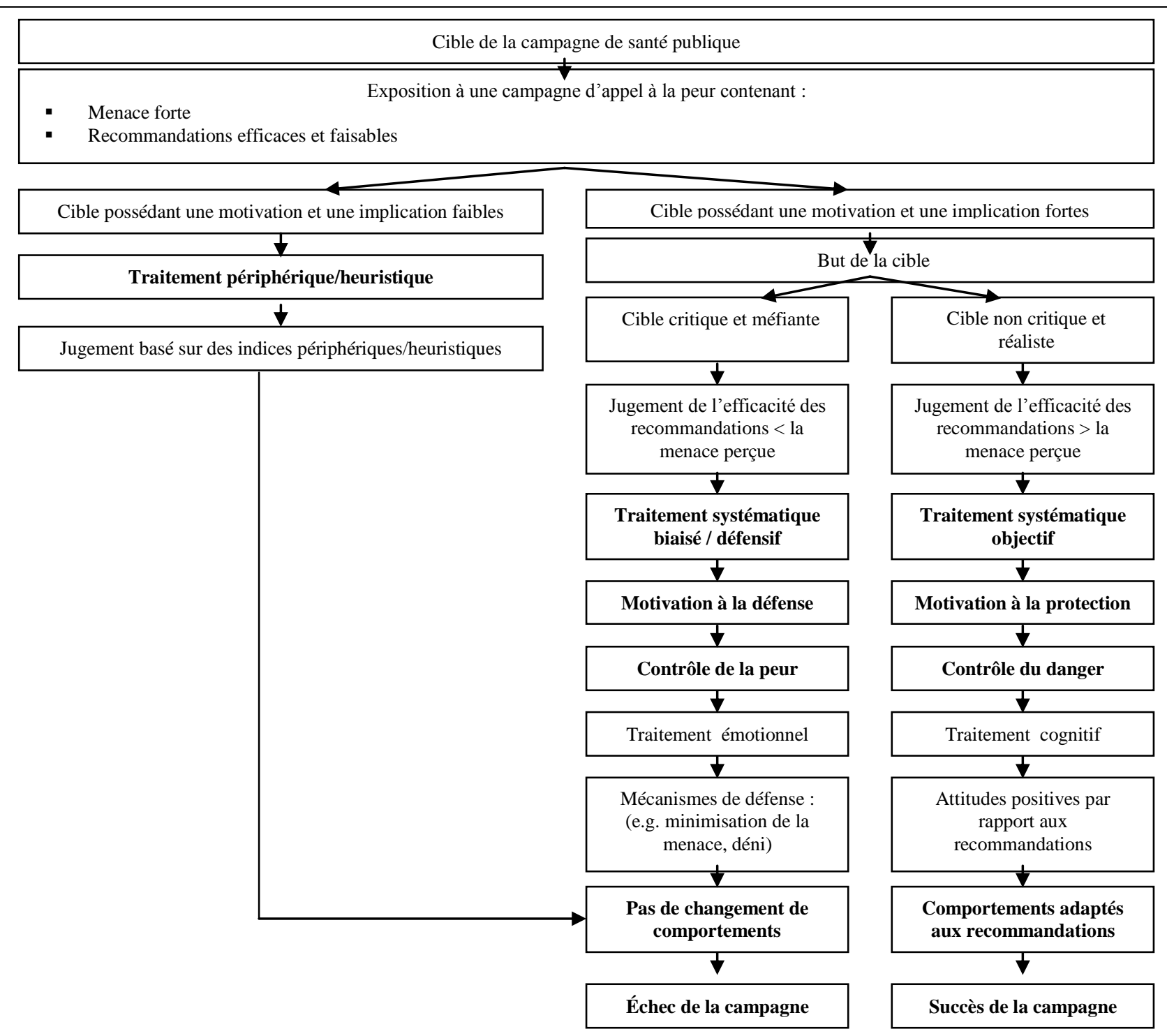

Figure 1- Un modèle schématique des appels à la peur en communication persuasive de santé publique 


\section{LA « COMMUNICATION ENGAGEANTE » : VERS UNE NOUVELLE FORME DE COMMUNICATION PERSUASIVE DE SANTE PUBLIQUE}

Le concept de «communication engageante » a été récemment introduit par Joule et al. (2004), il associe communication persuasive et théorie de l'engagement (Joule et Beauvois, 1998 ; 2002). Après une présentation de la communication engageante, nous montrons en quoi elle peut être une nouvelle forme de communication persuasive de santé publique et favoriser le changement des comportements de santé. Nous envisageons également ses implications pragmatiques pour le marketing social.

\subsection{De l'engagement à la persuasion : la « communication engageante »}

L'objectif principal des campagnes de marketing social dans le domaine de la santé est de changer les attitudes et les comportements. Si elles parviennent le plus souvent à modifier les attitudes des citoyens, modifier leurs comportements s'avère plus difficile. En effet, il n'est pas rare, qu'à la lecture d'un message de prévention, les récepteurs soient convaincus par les arguments du message mais ne changent pas leurs comportements pour autant. Par exemple, suite à la lecture d'un message contre le tabagisme, un fumeur peut être convaincu que l'arrêt du tabac est le seul moyen de diminuer les risques de cancer, mais continuer tout de même à fumer. Il y a un décalage entre les idées (les attitudes) et les actes (les comportements). Pour Joule et al. (2004), il suffit de « pas grand chose» pour passer des idées aux actes et ce «pas grand chose» serait un comportement engageant. Pour ces auteurs, si l'on veut persuader quelqu'un de réaliser un certain comportement, la requête finale doit être précédée par l'obtention d'un acte préparatoire allant dans le même sens. Par exemple, Joule (1987) voulait amener des fumeurs, ayant une importante consommation de tabac, à se priver de tabac pendant vingt heures (requête finale). Les résultats ont montré que les fumeurs amenés à s'abstenir pendant seulement deux heures (acte préparatoire engageant), acceptaient de se priver de tabac pendant vingt heures (changement comportemental). En revanche, ceux n'ayant pas été amenés à se priver de tabac pendant deux heures (non soumis à l'acte préparatoire) n'acceptaient pas de se priver de tabac pendant vingt heures. Ainsi, ce n'est pas parce que les fumeurs ont été persuadés qu'ils se conduisent différemment (e.g. ils acceptent de se priver de tabac pendant vingt heures), c'est parce qu'ils s'y sont engagés. Le mécanisme conduisant au changement de comportement repose sur une prise de décision (e.g. décision d'arrêter de fumer pendant deux heures) et le gel de cette décision, c'est-à-dire, au maintien coût que coût de la décision. Le principe de l'engagement entre dans la théorie psychosociale de la soumission librement consentie (Joule et Beauvois, 1998). L'engagement correspond «aux conditions de 
réalisation d'un acte qui, dans une situation donnée, permettent à un attributeur d'opposer cet acte à l'individu qui l'a réalisé» (op.cit. p. 60). Sont considérés comme attributeurs, les témoins oculaires, les acteurs eux-mêmes, mais également n'importe quelle personne qui aurait eu connaissance de ce qui s'est passé (Girandola, 2003). Il existe différentes procédures d'engagement ou de soumission librement consentie (voir Joule et Beauvois, 2002, pour une revue). Celle qui illustre le mieux le principe de comportement préparatoire engageant reste le pied-dans-la-porte. Son principe est simple : pour amener une personne à réaliser un comportement, on la prépare à se comporter d'une certaine façon, en lui faisant réaliser un acte anodin du même type et qui prépare le terrain.

Pour Joule et al. (2004), les techniques d'engagement peuvent être associées à la communication persuasive, c'est ce qu'ils nomment la «communication engageante ». Pour eux, une campagne persuasive (e.g. pour la prévention de la sécurité routière) a plus de chance d'être acceptée, si elle est précédée d'un acte préparatoire engageant consistant avec les arguments qu'elle développe (e.g. signature d'un bulletin d'engagement à ne pas conduire après avoir bu). Ainsi, la communication persuasive ne se résume plus dans la question : «qui dit quoi, à qui, dans quel canal et avec quel effet ? ». Mais la question est : «qui dit quoi, à qui, dans quel canal, en lui faisant faire quoi, et avec quel effet comportemental ? » (op. cit. p. 127). Pour Joule et al. (ibid.), si l'on recherche un effet comportemental, la question revient à chercher «quel est le comportement préparatoire engageant à obtenir du récepteur ? ». Ainsi, l'articulation entre engagement et persuasion donne naissance à un nouveau type de communication persuasive : la communication engageante. Celle-ci s'ancre plus généralement dans une forme de « communication-action » (ibid.) qui peut être mise au service des campagnes de marketing social pour changer les comportements de santé.

\subsection{Communication engageante et changement de comportements de santé : vers une nouvelle forme de communication persuasive en santé publique}

Envisageons, maintenant, l'application de la communication engageante en prévention de santé publique et ses effets sur le changement d'attitude et de comportement. La communication engageante agit à la fois à un niveau cognitif (attitudes) et à un niveau comportemental. Ses effets dépendent de la nature de l'acte préparatoire engageant. Celui-ci peut être problématique ou nonproblématique. Les actes problématiques sont contraires aux attitudes des individus (e.g. «je suis contre le dépistage du cancer ; je rédige un texte pour le dépistage du cancer ») ou leurs motivations (e.g. «j'aime fumer; je m'abstiens de fumer »). A l'inverse, les actes non-problématiques vont dans le sens des attitudes (e.g. «je suis pour le dépistage du cancer; je rédige un texte pour le dépistage 
du cancer ») et des motivations du sujet (e.g. «j'aime fumer, je fume »). En matière de santé publique, les professionnels cherchent à amener les citoyens à adopter des comportements contraires à leurs attitudes initiales (e.g. convaincre un fumeur d'arrêter de fumer), ils travaillent donc sur des actes préparatoires problématiques, c'est pourquoi nous nous limiterons à ceux-ci. Dans ce cas, la communication engageante aura pour effet, chez le récepteur, d'ajuster ses attitudes avec ses actes et de réaliser un nouveau comportement en accord avec ses actes. Ces effets s'expliquent en termes de dissonance cognitive et de rationalisation (Girandola, 2003). La réalisation d'un acte préparatoire problématique (e.g. s'abstenir de fumer pendant deux heures) crée un état de contradiction chez le sujet entre ses motivations (e.g. «j'aime fumer») et l'acte qu'il vient de réaliser (e.g. s'abstenir de fumer). Le sujet va chercher par tous les moyens à diminuer cet état de dissonance. Pour cela, il va ajuster a posteriori ses attitudes et ses comportements avec l'acte préparatoire. C'est ainsi, que le sujet va modifier ses attitudes et va réaliser d'autres actes en accord avec le comportement préparatoire (e.g. s'abstenir de fumer pendant une période plus importante). Le processus de rationalisation n'opère que si l'individu se sent libre de réaliser ou pas le comportement préparatoire (Joule et Beauvois, 1998). Ainsi, l'obtention du comportement préparatoire va optimiser l'acceptation de la campagne persuasive et ses effets. Par exemple, Stice et al., (2001) ont montré, dans le cadre de la prévention des désordres alimentaires chez les jeunes femmes, que celles exposées à un message persuasif après avoir effectué des comportements engageants (e.g., jeu de rôle contre la minceur comme idéal féminin) sont plus satisfaites de leurs corps et croient moins à la minceur comme idéal féminin (changement d'attitudes) que celles exposées uniquement au message persuasif. De plus, les effets perdurent quatre semaines après l'intervention. Notons, ici, un effet à long terme de la communication engageante (voir Girandola et Roussiau, 2003, pour une revue).

Nous voyons s'esquisser une nouvelle forme de communication persuasive en santé publique, dont nous sommes encore qu'aux prémices. S'il est avéré que l'impact d'une campagne persuasive en santé publique peut être optimisé lorsqu'elle est précédée d'un comportement préparatoire engageant, d'autres questions restent en suspens. Par exemple, comment le sujet ayant effectué un comportement préparatoire engageant va-t-il traiter le message persuasif (e.g. voie centrale vs. voie périphérique) ? Quels sont les facteurs (e.g. implication personnelle, perception du risque) pouvant moduler l'effet de la communication engageante ? De nombreuses recherches restent à mener dans ce domaine. C'est tout un nouveau champ de recherche combinant théories cognitives de la persuasion en santé publique et communication engageante qui s'ouvre. 


\subsection{Utiliser la communication engageante en marketing social : principes d'action et implications pour les praticiens}

La communication engageante favorise le changement de comportement en matière de santé, elle est donc d'un apport précieux pour le marketing social. Elle peut s'insérer au niveau du plan de campagne dans la stratégie de communication. Ainsi, la communication engageante permet d'allier communication média et hors média. Les campagnes de prévention médiatiques (e.g. presse, télévision, affichage) sont alors relayées sur le terrain par des actions hors-média (e.g. évènementiel, marketing direct) dont l'objectif est d'initier un comportement préparatoire engageant. Joule et al., (2004) relatent une recherche illustrant cette démarche. Ces auteurs ont cherché à promouvoir les économies d'énergie en utilisant la communication engageante et en l'insérant dans un plan média et hors média. Une campagne d'affichage avait été, tout d'abord, mise en place. Elle visait à inciter les citoyens à faire des économies d'énergie avec l'accroche suivante : «Pierre s'offre des CD en appuyant sur un interrupteur. Vous voulez économiser votre argent? Vous voulez préserver votre environnement? Alors faites comme Pierre appelez, nous! (suivait un numéro azur). La campagne d'affichage avait deux objectifs. D'une part, faire en sorte que le citoyen identifie son action : économiser de l'énergie, c'est aussi économiser de l'argent. D'autre part, elle encourageait les citoyens à effectuer un premier comportement engageant : appeler le numéro azur. La campagne d'affichage était assortie d'une démarche de marketing direct (e.g. mailing et accompagnement téléphonique). Les citoyens recevaient à domicile un test sur les économies d'énergie qui constituait un autre comportement engageant. Ils étaient, également, contactés par téléphone où des ingénieurs avaient pour objectif d'obtenir d'eux plusieurs comportements engageants, comme, répondre à des questions sur les économies d'énergie, aller retirer une brochure, faire une fiche familiale de diagnostic. L'évènementiel était utilisé sous forme d'un week-end de sensibilisation aux économies d'énergie au cours duquel les citoyens étaient invités à participer à des expositions, des conférences et à signer un bulletin d'engagement. Celui-ci était composé de deux parties : l'une destinée à être rendue et exposée dans les lieux publics, l'autre conservée par le signataire servant à la mémoire individuelle. Cet exemple montre comment la communication engageante peut s'insérer dans une démarche de marketing social et optimiser l'efficacité des actions.

Comment mettre en œuvre une telle démarche en marketing social et faire en sorte qu'un comportement devienne engageant? Voici quelques principes d'action. Un comportement préparatoire engageant peut prendre plusieurs formes : signer un bulletin d'engagement, répondre à un questionnaire, distribuer des tracs en faveur d'une cause. Mais pour que l'acte soit engageant, il 
doit être réalisé dans certaines conditions (Joule et Beauvois, 1998). Ainsi, il doit être effectué en public, du moins devant une instance attributive certifiant que l'acte a bien été réalisé par le sujet. L'acte doit avoir un caractère explicite (versus ambigu) et irrévocable. Il doit être répété (un acte réalisé plusieurs fois est plus engageant qu'un acte réalisé une fois), coûteux en temps et/ou en argent, et lourd de conséquences. De plus, le sujet ne doit pas réaliser l'acte à cause de raisons externes (e.g. promesse de récompense, menace de punition). Les raisons de l'acte doivent être internes pour que le sujet fasse le lien entre ce qu'il est et ses actes. Elles peuvent être favorisées par des auto-attributions internes, c'est-à-dire, des phrases du type «vous êtes vraiment quelqu'un de responsable ». Enfin, l'acte doit être réalisé dans un contexte de liberté, qui est le principal facteur d'engagement. L'individu doit croire qu'il est libre d'effectuer le comportement, cela passe par l'utilisation de phrase du type «c'est à vous de voir, je comprends très bien que vous refusiez ». En résumant la méta-analyse de Burger (1999), un comportement est engageant s'il a été effectivement réalisé dans un contexte de liberté, s'il a un certain coût et n'est pas lié à une compensation financière ou une promesse de récompense. Le comportement préparatoire et le comportement final doivent relever de la même identification de l'action (e.g. s'abstenir de fumer pendant deux heures (comportement préparatoire) pour s'abstenir pendant 20 heures (comportement final)) et ne doivent pas être formulés par la même personne. En appliquant ces principes, les professionnels du marketing social peuvent augmenter l'impact de leurs campagnes.

\section{QUELQUES IMPLICATIONS PRATIQUES POUR LES PROFESSIONNELS DU MARKETING SOCIAL}

D'un point de vue opérationnel, les recherches en communication persuasive de santé publique possèdent des implications pratiques pour les professionnels du marketing social, principalement au niveau de la connaissance et la segmentation de la cible, de la conception des campagnes et du plan de communication. Voici quelques conseils destinés aux praticiens du marketing social afin d'accroître l'efficacité des campagnes.

\subsection{Connaissance et segmentation de la cible}

Afin d'optimiser l'impact de leurs démarches, les professionnels du marketing social veilleront à sélectionner une cible impliquée par le thème de la campagne et motivée à se protéger de la menace. Il est également conseillé de définir une cible possédant de nombreuses connaissances sur le thème de la campagne, car elle est plus sensible à la qualité des arguments d'un message (Wood et al, 1995). Les comportements antérieurs de la cible sont des indices que les professionnels du marketing social doivent prendre en compte. Plus une cible a l'habitude d'effectuer 
quotidiennement un comportement (e.g. conduire sans ceinture de sécurité), plus il sera difficile de le modifier, car il relève d'un automatisme (Girandola, 2003). Connaître les comportements antérieurs de la cible, permet aux praticiens d'adapter leurs messages. Le besoin de cognition ou appétence pour l'effort cognitif de la cible est également un indice pertinent. Une cible possédant un fort besoin en cognition sera plus influencée par la qualité des arguments du message. En revanche, une cible ayant un faible besoin en cognition sera plus influencée par des messages émotionnels (e.g. humoristiques). Les professionnels du marketing social veilleront également à identifier si le comportement mis en cause par la campagne fait partie de l'image de soi (e.g. fumer). Plus le comportement fait partie de l'image de soi, plus il est difficile à modifier car sa modification remet en cause l'identité de la cible (Courbet, 2003). Pour préserver son identité, la cible rejettera la campagne.

\subsection{Conception des campagnes et des messages}

Pour concevoir des campagnes efficaces, les professionnels du marketing social peuvent utiliser les appels à la peur en ayant recours à des images vivides (e.g. utilisation de photographies en couleur montrant une tumeur cancéreuse) et à un vocabulaire cru. Cependant, pour éviter un effet « boomerang », les messages d'appel à la peur doivent toujours comporter des recommandations efficaces et faisables afin de diminuer la vulnérabilité et d'augmenter le sentiment d'auto-efficacité (Witte, Allen, 2000). Pour augmenter la perception de l'efficacité des recommandations, les professionnels du marketing social doivent montrer clairement en quoi les recommandations sont efficaces pour diminuer le risque (e.g. utilisation de statistiques montrant que les personnes ayant arrêté de fumer ont diminué leur risque de contracter un cancer). L'impact des recommandations peut être optimisé par l'utilisation d'un plan d'action (e.g. prendre rendez-vous avec un professionnel de la santé, appeler un numéro vert). Les appels à la peur ne sont efficaces que pour promouvoir un comportement de prévention (e.g. se protéger lors de l'exposition au soleil). Si l'objectif de la campagne est de promouvoir un comportement de détection (e.g. effectuer un dépistage du cancer de la peau), un message générant une faible peur sera plus efficace (Girandola et Atkinson, 2003). Les campagnes ne doivent pas remettre en doute la confiance de la cible sur ses connaissances par rapport au risque en utilisant des formules du type : «Vous avez un doute ? Sida Info service, des réponses pour y voir plus clair ». Elles engagent un effet indésirable dans la manière dont les individus abordent les connaissances nouvelles de la campagne, évaluent la menace et les moyens pour y faire face (Terrade et Meyer, 2003). L'efficacité des campagnes peut être optimisée par l'utilisation d'un vocabulaire personnalisé (e.g. «vous pouvez contracter un cancer ») et de questions (e.g. «n'est-ce pas une bonne idée d'utiliser des préservatifs ?»). Ces 
figures de rhétorique permettent d'augmenter le niveau d'implication de la cible (Girandola, 2002). D’une manière générale, pour augmenter l'efficacité des campagnes, les professionnels du marketing social doivent concevoir des messages «taillés sur mesure » en fonction de la cible (op.cit). Par exemple, le cadrage du message est fonction de l'implication de la cible (Girandola, 2000). Si la cible est fortement impliquée par le thème du message, un cadrage négatif présentant l'inconvénient d'un rejet des recommandations (e.g. « Si vous n'arrêtez pas de fumer, vous augmentez votre risque de contracter un cancer ») est plus efficace. En revanche, si la cible n'est pas impliquée par le thème de la campagne, un cadrage positif présentant l'avantage d'adopter les recommandations (e.g. «Si vous arrêtez de fumer, vous diminuez votre risque de contracter un cancer ») est plus persuasif. Le type de message dépend également de l'implication de la cible. Un message émotionnel basé sur un témoignage (e.g. un individu raconte comment il a contracté le VIH) est plus efficace quand la cible est faiblement impliquée. Un message rationnel utilisant des statistiques est plus efficace quand la cible est fortement impliquée.

\section{3. Élaboration du plan de communication}

Les recherches en communication persuasive de santé publique permettent également de donner des recommandations aux professionnels du marketing social au niveau du plan de communication. Ainsi, il est conseillé d'utiliser un mix-communication et de multiplier les moyens de communication média et hors-média. Afin d'augmenter l'impact des campagnes de marketing social, les praticiens veilleront à répéter leurs messages. L'efficacité optimum est constatée lorsque le message génère une forte peur et qu'il est répété au moins cinq fois (Courbet, 2003). La communication engageante montre l'intérêt pour les professionnels du marketing social d'associer campagnes persuasives médiatiques et techniques d'engagement. Ainsi, pour une meilleure efficacité, les campagnes de prévention peuvent être alliées à des actions engageantes sur le terrain via des moyens de marketing direct (e.g. mailing accompagnement téléphonique) ou de communication évènementielle (e.g. organisation de journée de prévention permettant la signature d'un acte engageant).

\section{CONCLUSION}

S'intégrant dans la thématique générale de ce numéro spécial, l'article a permis d'illustrer un domaine d'application de la communication persuasive au marketing: la prévention de santé publique. Au travers d'une revue des récents modèles de la communication persuasive de santé publique, il a mis en évidence l'apport important de cette discipline au marketing social. Ainsi, les 
modèles cognitifs du changement de comportement de santé, les modèles des «appels à la peur » et le nouveau concept de communication engageante ont permis, d'une part, d'expliquer les effets des campagnes de marketing social sur le changement de comportement et, d'autre part, de donner lieu à des implications pragmatiques pour les praticiens. L'application de la communication persuasive au marketing social offre de belles perspectives de recherches. Une d'entre elles repose sur la communication engageante. D'une part, il s'agirait de coupler ce concept avec les modèles des traitements des messages de prévention (e.g. modèles duaux de la persuasion, modèles des appels à la peur) afin d'identifier, à la fois le rôle du comportement préparatoire engageant dans les traitements et l'effet de son interaction avec des variables propres à la prévention de santé (e.g., motivation à la défense, perception du risque). D'autre part, il s'agirait d'opérationnaliser ce concept en l'intégrant à la démarche du marketing social afin d'augmenter l'impact des campagnes. L'intérêt étant d'associer communication persuasive et marketing social dans un même but: la prévention en matière de santé.

\section{RÉFÉRENCES}

- Ajzen, I, (1991), «The theory of planned behavior », Organizational Behavior and Human Decision Processes, 50, 179-211.

- Albarracín, D., Johnson, B.T., Fishbein, M., Muellerleile, P.A., (2001), « Theories of reasoned action and planned behavior as models of condom use: a meta-analysis », Psychological Bulletin, 127, 142-161.

- Arthur, D., Quester, P., (2004), «Who's afraid of that ad? Applying segmentation to the protection motivation model », Psychology \& Marketing, 21 (9), 671-696.

- Baron, R., Logan, H., Lilly, J., Inman, M., Brennan, M., (1994), « Negative emotion and message processing », Journal of Experimental Social Psychology, 21, 181-202.

- Burger J. M, (1999), «The foot-in-the-door compliance procedure: a multiple-process analysis and review », Personality and Social Psychology Review, 3, 303-325.

- Chabrol, C., Courbet, D., Fourquet-Courbet, M.P., (2004), « Psychologie sociale, traitements et effets des médias », Questions de Communication, 5, 5-19.

- Chaiken, S., Trope, Y., (1999), Dual-process theories in social psychology, NY, The Guilford Press.

- Chaiken, S, Giner-Sorolla, R, Chen, S, (1996), «Beyond accuracy: defense and impression motives in heuristic and systematic information processing» in Gollwitzer, P. M., Bargh, J. A., (Eds) The psychology of action: linking cognition and motivation to behavior, NY, The Guilford Press, 553-578.

- Chaiken, S., Liberman, A., Eagly. A.H., (1989), «Heuristic and systematic information processing within and beyond the persuasion context » in Uleman J.S., Bargh J.A., (eds), Unintended Thought, NY, Guilford Press, 212-252.

- Chen, S., Chaiken, S., (1999), « The heuristic-systematic model in its broader context » in Chaiken, S., Trope, Y., Dual-process theories in social psychology, NY, The Guilford Press, 73-96.

- Corneille, O., (1993), «Une synthèse critique du modèle de la probabilité d'élaboration », L'Année Psychologique, 93, 4, 583-602.

- Courbet, D., (2004), «L'influence non consciente des publicités vues furtivement et aussitôt oubliées. Une méthode d'étude socio-cognitive appliquée à l'Internet », Questions de Communication, 5, 83-102. 
- Courbet D., (2003), «Réception des campagnes de communication de santé publique et efficacité des messages générant de la peur. Une étude expérimentale sur le rôle de la peur dans le changement d'attitudes néfastes », Communication, 22 (1), 100-122.

- Das, E., De Wit, J.B., Stroebe, W., (2003), «Fear appeals motivate acceptance of action recommendations: evidence for a positive bias in the processing of persuasive messages », Personality and Social Psychology Bulletin, 29 (5), 650-664.

- Gallopel K., (2003), «Le marketing social: un outil au service de la prévention des comportements tabagiques des jeunes français ", in Prévention et prise en charge du tabagisme chez les étudiants, ouvrage collectif du Groupe d'expertise collectif tabac, éditions INSERM, 181-194.

- Girandola, F., (2003), Psychologie de la persuasion et de l'engagement, Besançon, Presses Universitaires de Franche-Comté.

- Girandola, F., (2002), «Persuasion et santé publique » in Bonardi C., Girandola, F., Roussiau N., Soubiale N., (dirs.), Psychologie Sociale Appliquée : environnement, santé, qualité de vie, Paris, In Press, 121-139.

- Girandola, F., (2000), «Peur et Persuasion : Présentation des recherches (1953-1998) et d'une nouvelle lecture », L’Année Psychologique, 100, 2, 333-376.

- Girandola, F., Roussiau, N., (2003), «L'engagement comme source de modifications à long terme», Cahiers Internationaux de Psychologie Sociale, 57 (1), 83-101.

- Girandola F., Atkinson D., (2003), «Prévention, détection et traitement de l'information persuasive en situation de peur », Revue canadienne des sciences du comportement, 35 (3), 197-208.

- Godin, G., (2002), «Le changement des comportements de santé » in Fischer G.N., (dir), Traité de psychologie de la santé, Paris : Dunod, 375-388.

- Griffin R. J, Dunwoody S; Neuwirth K., (1999), «Proposed model of the relationship of risk information seeking and processing to the development of preventive behavior», Environmental Research, 80, 230-245.

- Higgins, A.; Conner, M., (2003), «Understanding adolescent smoking: The role of the Theory of Planned Behaviour and implementation intentions », Psychology, Health \& Medicine, 8(2), 173-186.

- Joule, R.V., (1987), «Tobacco deprivation: the foot-in-the-door technique versus the low-ball technique », European Journal of Social Psychology, 17, 361-365.

- Joule, R.V., Beauvois, J.L., (2002), Petit traité de manipulation à l'usage des honnêtes gens, Grenoble : Presses Universitaires de Grenoble.

- Joule, R.V., Beauvois, J.L., (1998), La soumission librement consentie, Paris : Presses Universitaires de France.

- Joule R.V. Py J., Bernard F., (2004), «Qui dit quoi, à qui, en lui faisant faire quoi ? Vers une communication engageante », in Bromberg M, Trognon A (dirs), Psychologie sociale et communication, Paris : Dunod, 205-218.

- Kotler P., Roberto N., Lee N., (2002), Social Marketing: Improving the Quality of Life, Sage Publication.

- Kreuter, M. W., Bull, F. C., Clark, E. M., \& Oswald, D. L. (1999). «Understanding how people process health information. A comparison of tailored and non-tailored weight-loss materials », Health Psychology, 18 (5), 487-494

- Meyer T. (2000) «Le modèle de traitement heuristique/systématique de l'information : motivations multiples et régulation du jugement en cognition sociale », L'Année Psychologique, 100, 3, 527-563.

- Petty R. E., Wegener D.T., (1999), «The elaboration likelihood model: current status and controversies », in Chaiken, S., Trope, Y., (1999), Dual-process theories in social psychology, NY, The Guilford Press, 137-165.

- Petty R.E., Cacioppo J.T., (1986), «The Elaboration Likelihood Model of Persuasion », in Berkowitz, L. (Ed), Advances in Experimental social Psychology, vol 19, Academic Press, 123-205. 
- Prochaska, J.O., DiClemente C.C., Norcross, J.C., (1992), « In search of how people change: application to addictive behaviors », American Psychologist, 47, 1102-1114.

- Rogers R. W, (1983), «Cognitive and physiological processes in fear appeals and attitude change: a revised theory of protection motivation », in Cacioppo J., Petty R., Social psychophysiology, N.Y, Guildford, 153-176.

- Rosen, C.S.; (2000), «Integrating stage and continuum models to explain processing of exercise messages and exercise initiation among sedentary college students », Health Psychology, 19(2), 172-180.

- Ruiter R., Abraham C., Kok G., (2001), «Scary warnings and rational precautions: a review of the psychology of fear appeals », Psychology and Health, 16, 613-630.

- Schoenbachler D.D, Whittler T E , (1996), «Adolescent processing of social and physical threat communications », Journal of Advertising, 25, 37-54.

- Schuette, R.A., Fazio, R.H., (1995), «Attitude accessibility and motivation as determinants of biased processing: a test of the MODE model », Personality and Social Psychology Bulletin, 21, 704-710.

- Stephenson, M.T., Witte, K. (1998). «Fear, threat, and perceptions of efficacy from frightening skin cancer messages », Public Health Reviews, 26, 147-174.

- Stice E., Chase A. Stormer S., Appel A., (2001), «A Randomized Trial of a Dissonance-Based Eating Disorder Prevention Program », International Journal of Eating Disorders, 29 (3), 247-262

- Sturges JW, Rogers RW, (1996), «Preventive health psychology from a developmental perspective: an extension of protection motivation theory », Health Psychology, 15, 158-166.

- Terrade F., Meyer T., (2003), «Auto-évaluation des connaissances et élaboration défensive de l'information sur le risque : application à la lecture d'un message sur le sida », Cahiers Internationaux de Psychologie Sociale, 59, 40-53.

- Witte K. (1992), «Putting the fear back into fear appeals: The extended parallel process model », Communication Monographs, 59, 329-349.

- Witte K. (1998), «Fear as motivator, fear as inhibitor: Using the extended parallel process model to explain fear appeals successes and failures », in Andersen P. A, Guerrero L K (Eds), Handbook of communication and emotion, Academic Press, 423-450.

- Witte K., Allen, M., (2000), «A meta-analysis of fear appeals: implications for effective public health campaigns », Health Education and Behaviour, 27, 591-615.

- Witte K. Donohue, W.A., (2000), «Preventing vehicle crashes with trains at grade crossings: the risk seeker challenge », Accident Analysis and Prevention, 32, 127-139.

- Wood, W., Rhodes N., Biek, M., (1995), «Working knowledge and attitude strength: an information processing analysis », in Petty R.E., Krosnick J.A., (Eds), Attitude strength: antecedents and consequences, Mahwah, NJ, Erlbaum, 283-313. 